\title{
Relevance of Cooperative Lattice Effects and Correlated Disorder in Phase-Separation Theories for CMR Manganites
}

\author{
Jan Burgy, Adriana Moreo and Elbio Dagotto ${ }^{1}$ \\ ${ }^{1}$ National High Magnetic Field Lab and Department of Physics, Florida State University, Tallahassee, FL 32306
}

(Dated: November 8, 2018)

\begin{abstract}
Previous theoretical investigations of colossal magnetoresistance (CMR) materials explain this effect using a "clustered" state with preformed ferromagnetic islands that rapidly align their moments with increasing external magnetic fields. While qualitatively successful, explicit calculations indicate drastically different typical resistivity values in two- and three-dimensional lattices, contrary to experimental observations. This conceptual bottleneck in the phase-separated CMR scenario is resolved here considering the cooperative nature of the Mnoxide lattice distortions. This induces power-law correlations in the quenched random fields used in toy models with phase competition. When these effects are incorporated, resistor-network calculations reveal very similar results in two and three dimensions, solving the puzzle.
\end{abstract}

PACS numbers: PACS numbers: 75.47.Lx, 75.30.Kz, 75.50.Ee, 75.10.-b

The study of self-organization in transition-metal oxides is one of the dominant scientific themes of condensed matter physics (CMP). This phenomenon includes the intrinsically inhomogeneous states of CMR manganite ${ }^{1,2}$ and underdoped high temperature superconductors ${ }^{3}$. In these compounds, the competition between different ordering tendencies leads to complexity: their properties change dramatically upon the application of relatively small perturbations. In Mnoxides, the cross-fertilization between theoretical and experimental investigations has been remarkably fruitful, and at present the existence of mixed-phase tendencies in the CMR regime is widely accepted ${ }^{4}$. The emerging CMR picture is based on nanoscale clusters of competing phases ${ }^{2.45 .6}$. With increasing magnetic fields, the clusters with ferromagnetic (FM) characteristics rapidly align their moments, leading to a percolative insulator-metal transition. Several other compounds share similar phenomenology, and "clustered states" are rapidly emerging as a novel paradigm of $\mathrm{CMP}^{7}$.

To our knowledge, the only major unresolved issue that confronts the phase-separation scenario for the CMR oxides concerns the dimensionality dependence of current theoretical descriptions. Recent resistor-network calculations in two dimensions (2D) reported a colossal MR effect, compatible with experiments, near the clean-limit first-order FMantiferromagnetic (AF) phase transition. Disorder was further shown to smear the FM-AF transition region into a glassy clustered state ${ }^{6}$. Well-known arguments ${ }^{\frac{8}{-}}$ predicts that, in $2 \mathrm{D}$, infinitesimal disorder is sufficient to create large coexisting clusters of neighboring phases, due to the competition between cluster surface effects and the random impurity distribution inside a bubble of one phase embedded into another. However, similar simulations in three dimensions (3D) (shown below) do not lead to equally impressive resistivity $\rho$ vs. temperature curves. Within the Imry-Ma reasoning $\frac{8}{8}$ the critical dimension is 2 , and only an unphysically large disorder strength $\Delta_{c}$ can destabilize the uniform 3D FM phase of the Random Field Ising Model (RFIM). Moreover, for $\Delta \geq \Delta_{c}$ the resulting clusters are not large enough to induce a substantial $\rho$. Therefore, it is crucial to resolve this incorrect dimensional dependence ${ }^{9}$. Since the phenomenology emerg- ing from computer simulations in 2D matches qualitatively the experimental results gathered in both $2 \mathrm{D}$ and $3 \mathrm{D}$, mixedphase tendencies likely dominate in real materials. Moreover, recent experiments have unveiled a remarkable instability of the CE phase to the introduction of disorder in 3D, showing that disorder is by no means irrelevant in real perovskite manganites 10 . This is also compatible with recent small-clusters simulations ${ }^{11,12}$. Nevertheless, in the transition from realistic models, which cannot be simulated on large enough lattices to reach percolation, to the RFIM-like toy models that can successfully estimate magnetoresistances ${ }^{6}$, the appearance of an unphysical dimensionality dependence suggests that an important conceptual ingredient has been lost.

In this paper, the dimensionality-dependence puzzle is solved. The crucial issue unveiled here is the key relevance of cooperative effects for quantitative magnetoresistance studies of Mn-oxides. Cooperation introduces correlations in the quenched disorder needed to render percolative the clean-limit standard FM-AF first-order transition of simple models of phase competition. Previous simulations used uncorrelated disorder ${ }^{6}$, and this induced the substantial quantitative differences between $2 \mathrm{D}$ and $3 \mathrm{D}$. The disorder discussed here is inevitable - and, thus, intrinsic - in the standard chemicaldoping process widely used to control the hole density, or average hopping amplitude, in transition-metal oxides. Replacing tri- by di-valent ions of different sizes, introduces $\mathrm{MnO}_{6}$ octahedra distortions that cause local disorder. Once a distortion is created at a given lattice site, this distortion propagates following a power-law decay $1 / r^{\alpha}$ governed by standard elasticity mechanisms that suggest $\alpha \sim 3$ (for recent literature see Refs 14 15). This propagation emerges from the cooperative nature of the distortions, since adjacent $\mathrm{MnO}_{6}$ octahedra share an oxygen. This cooperation was already shown ${ }^{16}$ to be crucial for understanding the charge-order states at commensurate fillings, such as $x=0.5$. The present effort shows that cooperation is also crucial for the understanding of the dimensionality dependence of CMR simulations.

The relevance of elastic effects has already been emphasized ${ }^{15}$ using Ginzburg-Landau free energies. Elastic compatibility constraints were found to generate texture- 

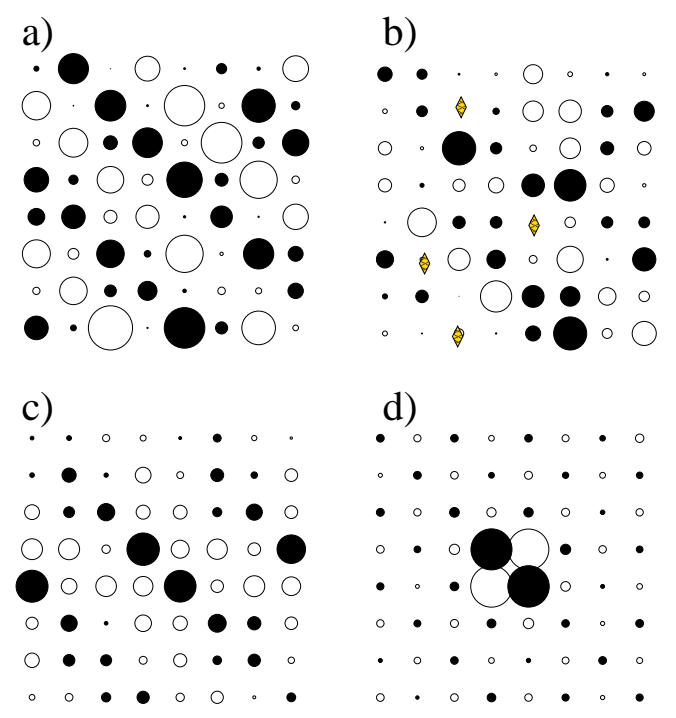

FIG. 1: MC results for the two-orbital DE model with cooperative JT phonons, on a $8 \times 8$ lattice at low temperature $(T)$. The Hund coupling is $\infty$, and the classical spins are assumed ferromagnetically aligned for simplicity. Cooperation is included by using the oxygen coordinates as d.o.f. Only the $\mathrm{Q}_{2}$ mode is active (not a restrictive assumption, since at $x=0$ and $0.5, \mathrm{Q}_{2}$ is the most relevant mode ${ }^{2}$ ). (a) Clean-limit results for $\lambda=1.6>\lambda_{c}=1.4$, and $x=0.5$. The dark and open circles indicate positive and negative $\left\langle\mathrm{Q}_{2}\right\rangle$, respectively, concomitant with populated orbitals oriented along the $x$ - and $y$-axis. The $\left\langle\mathrm{Q}_{2}\right\rangle$ absolute value, proportional to the dot radius, is related to the charge at each site. The previously documented stripes ${ }^{1,2,4}$ are clearly observed in the simulation (the small deviations from a perfect arrangement are caused by finite- $T$ effects). (b) Same as (a) but with 4 sites (diamonds) where $\lambda=0.0$. Now the stripe pattern is drastically disrupted, showing the high sensitivity of the $\mathrm{CO}$ state to disorder $^{11,12}$. (c) Illustration of the opposite effect as in (a-b): here $\lambda$ is subcritical $(=1.2)$ everywhere but in the 4 sites with the largest dots where $\lambda=2>\lambda_{c}$. A clean-limit study with uniform $\lambda=1.2$ reveals no order, but the inclusion of just 4 sites with $\lambda>\lambda_{c}$ clearly creates short-range stripe order. Here and in (d) the dot area is proportional to $\left\langle\mathrm{Q}_{2}\right\rangle$. (d) Similar as (c) but for $x=0.0 . \lambda=0.4$ (below $\lambda_{c}=0.5$ ) at all sites but the 4 with the largest dots, where $\lambda=2.0$. The ordered plaquette generates charge-ordering on the entire 64-site lattice.

inducing anisotropic long-range elastic forces, similar to those appearing in several transition-metal oxides. Strain effects have been discussed in manganite thin films as wel1 ${ }^{18}$, and they could play a role in stabilizing charge-ordered states 19 . The effect of long-range Coulomb interactions near first-order transitions has also been recently investigated by Yang ${ }^{20}$, who found an interesting dependence of critical dimensions with the interaction range.

The key relevance of cooperative effects can be dramatically illustrated via Monte Carlo (MC) simulations of the two-orbital double-exchange (DE) model coupled to JahnTeller (JT) classical phonons. The Hamiltonian and details of the simulations have been extensively described in previ-
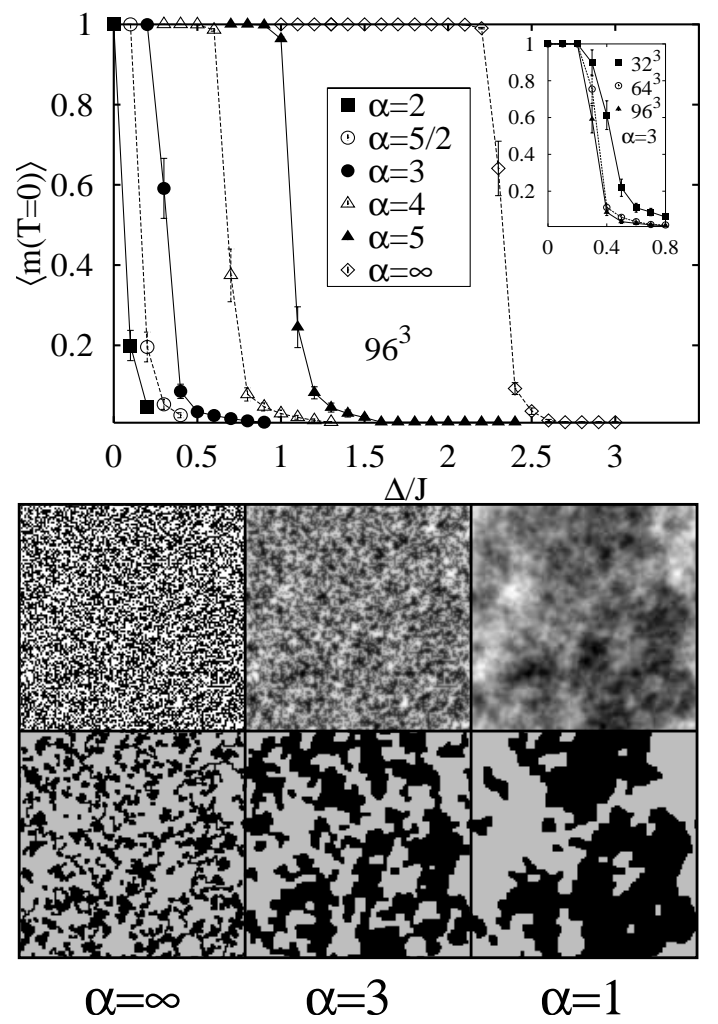

FIG. 2: Top panel: Computer-generated ground-state magnetization $\langle m(T=0)\rangle$ of the RFIM vs. $\Delta$, for several values of the disorder correlation exponent. $\alpha=2.5$ is the predicted 3D critical value below which infinitesimal disorder will destroy long-range order. Note the dramatic difference among the several $\alpha$ s. The inset shows $\langle m(T=0)\rangle$ vs. $\Delta$ for three different lattice sizes and $\alpha=3$, to illustrate finite-size effects. Bottom panel: Snapshots of a typical random field distribution $\tilde{h}_{i}$ (top row) and corresponding Ising spin configuration (bottom), for three $\alpha$ s on a $128^{2}$ lattice and $\Delta / J=1$, to visualize cluster sizes and shapes. 2D clusters are used to access large linear sizes, but results are similar in 3D (see Fig 3 , $)^{13}$.

ous literature ${ }^{16}$ and they will not be repeated here. The explicit use of oxygen degrees of freedom (d.o.f.) introduces cooperation in the distortions. To simplify the calculation and allow the study of 64-site clusters, the $t_{2 \mathrm{~g}}$ classical spins were frozen in a FM state and the transitions studied here only involve the charge/orbital d.o.f. This is certainly not restrictive since recent studies $\stackrel{11}{\underline{ }}$ have unveiled charge/orbital orderdisorder transitions at hole-density $x=0.5$ even with the spins in a FM configuration, by varying the electron-phonon coupling $\lambda$. The charge-ordered (CO) phase has the same arrangement of charge and orbitals as the realistic CE state ${ }^{11,17}$. Although the MC study in Fig 1 is necessarily restricted to $2 \mathrm{D}$, this is sufficient to show the key role of oxygen cooperation, illustrating the limitations in previous uncorrelateddisorder assumptions. Typical results in the $x=0.5$ clean-limit, and with $\lambda$ larger than the critical value $\lambda_{c}$ toward a COstate ${ }^{11}$, reveal the familiar pattern of charge-diagonal stripes with $\left(3 x^{2}-r^{2} / 3 y^{2}-r^{2}\right)$ populated orbitals (Fig 1 $)$. This order is dramatically affected when, to simulate quenched disor- 
der, the value of $\lambda$ is made subcritical in 4 sites of the 64site cluster (just $\sim 6 \%$ of the sites, Fig 1b). The stripe pattern disappears and a random-looking distribution of charge and orbitals is stabilized, due to the non-local character of the disturbance caused by the 4 subcritical sites (compatible with recent simulations ${ }^{11,12}$ ). In Fig 1, the situation is reversed: the background has a $\lambda<\lambda_{c}$ and, as a consequence, the lowest-energy state is not charge/orbital ordered in the clean limit. However, once 4 sites carry $\lambda>\lambda_{c}$, a stripe-like pattern emerges, affecting most of the lattice. Finally, even at $x=0$, having the 4 sites of a plaquette above $\lambda_{c}$ - with the rest below $\lambda_{c}$ (see Ref 22) - is sufficient to induce a staggered pattern on the entire cluster (Fig 1 $\mathrm{d}$ ). These realistic-model simulations clearly show that cooperation dramatically enhances the role of quenched disorder in manganite models.

The results in Fig 1 indicate that it is inappropriate to use the RFIM with uncorrelated disorder to mimic the physics of Mn-oxides. If a chemical-doping-induced lattice distortion at a Mn-Mn link leads to, e.g., a decrease of the hopping amplitudes, the neighboring links tend to have a similar reduction due to the slow power-law decrease of the elastic distortion. As a consequence, a proper RFIM modeling of real manganites requires a correlation in the random fields. More formally, consider the modified RFIM Hamiltonian

$$
H=-J \sum_{\langle i j\rangle} s_{i} s_{j}-\Delta \sum_{i, j} h_{i} s_{j} / d_{i j}^{\alpha},
$$

where $s_{i}$ are Ising variables, $J$ is the FM coupling, $\Delta$ is the disorder strength, and $d_{i j}$ is the distance between lattice sites $i$ and $j$ (in practice, $d_{i j}^{\alpha}$ was replaced by $\left(1+d_{i j}^{2}\right)^{\alpha / 2}$, with the same asymptotic behavior but remaining finite at zero distance). In this model, a 'random' perturbation $h_{i}$ at site $i$, influences the neighboring dynamical variables $s_{j}$ well beyond the usual on-site $i=j$ range, as the analysis in Fig 1 indicates. By redefining, $\tilde{h}_{j}=\sum_{i} h_{i} / d_{i j}^{\alpha}$, the Hamiltonian Eq1 can be cast in the standard form $H=-J \sum_{\langle i j\rangle} s_{i} s_{j}-\Delta \sum_{j} \tilde{h}_{j} s_{j}$, but now with correlated disorder since $\left\langle\tilde{h}_{i} \tilde{h}_{j}\right\rangle=1 / d_{i j}^{2 \alpha-D}(D=$ lattice dimension). The critical value of $\alpha-$ below which the system breaks into domains for infinitesimal $\Delta-$ is $\alpha_{c}=(D / 2)+1$, which for $D=3$ is $\alpha_{c}=2.5$ (for details, the reader should consult previous literature on correlated disorder such as Ref 21, and references therein). The important point for our purposes is that correlated disorder can alter the critical dimension, and its value can be raised to 3 if $\alpha \leq 2.5$. To test these ideas, model Eq1 1 has been studied using algorithms that allows for the direct calculation of RFIM ground states $^{23}$ (see Fig 2). In agreement with our expectations, there is a dramatic qualitative difference between the results obtained with uncorrelated disorder $(\alpha=\infty)$, and those obtained using a value of $\alpha(\alpha=3)$ that more realistically mimics the elasticity. In particular, Fig 2 shows that the former exhibits a large $\Delta_{c}$ and small clusters, while the latter has large clusters and a $\Delta_{c} \sim 10$ times smaller than the value obtained with uncorrelated disorder.

To further verify these ideas, the formalism presented in Ref 6 is here employed. To generate phase competition, a simple spin model with competing interactions is
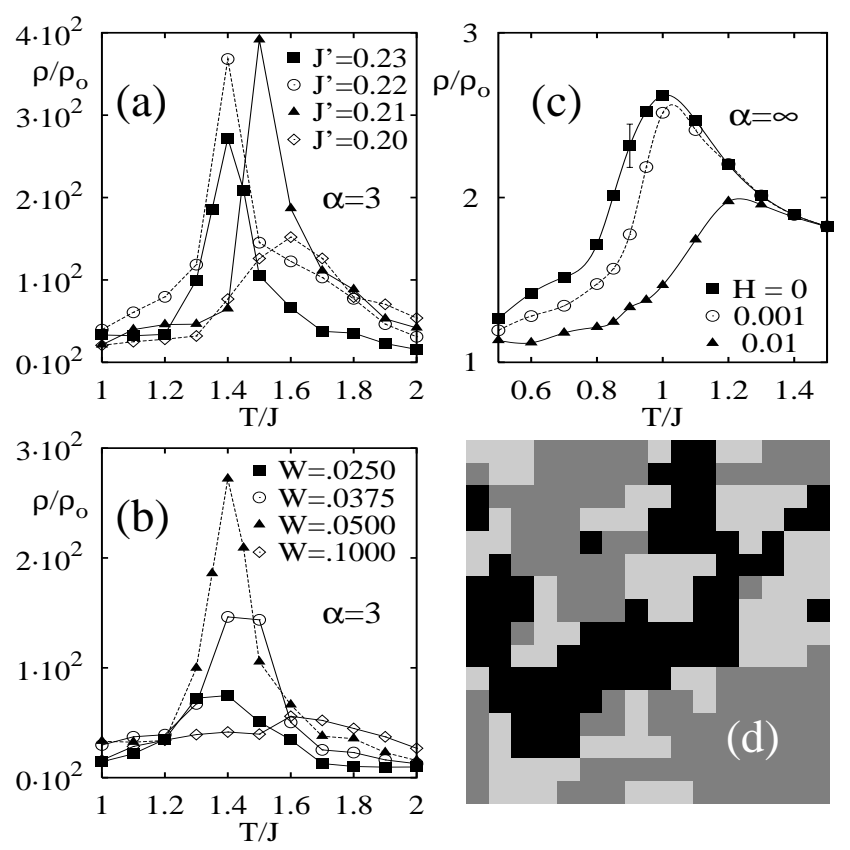

FIG. 3: (a) $J^{\prime}$ dependence of $\rho$ (in units of the metallic regions resistivity $\rho_{0}$ ) for the $3 \mathrm{D} J-J^{\prime}$ model with long-range correlated disorder ( $\alpha=3$ ), at $W / J=0.05$. (b) $\rho$ dependence on the disorder strength $W$, at $J^{\prime} / J=0.23$. (c) $\rho$ in $3 \mathrm{D}$ without long-range correlated disorder (i.e. at $\alpha=\infty)$. Note the small value of $\rho / \rho_{0}$ compared with (a,b). (d) Slice of a $32^{3}$ lattice at $T / J=1.4, J^{\prime} / J=0.23$ and $W / J=0.05$. Black and dark-gray represent FM regions with opposite orientations of their magnetic moments, and light-gray represents competing state (collinear AF) regions.

used $H=-J \sum_{\langle i j\rangle} s_{i} s_{j}+J^{\prime} \sum_{[i k]} s_{i} s_{k}$, where $s_{i}$ are Ising variables, and $J\left(J^{\prime}\right)$ is a nearest-neighbors (next-nearestneighbors) FM (AF) coupling. For small $J^{\prime} / J$, the $T=0$ dominant state is FM, while at large $J^{\prime} / J$ it has collinear AF features (alternating lines of spins up and down). The clean-limit critical value is $J^{\prime} / J=0.25(0.5)$ in $3 \mathrm{D}$ (2D). Disorder is introduced by the replacement $J^{\prime} \rightarrow J_{i k}^{\prime}=J^{\prime}+W \tilde{\epsilon}_{i k}$ at every plaquette diagonal, with $\tilde{\epsilon}_{i k}$ being random numbers in $[1 / 2,-1 / 2]$ spatially correlated as $\tilde{h}_{j}$. Disorder reduces the values of the clean-limit critical temperatures $T^{*}$ to $T_{C}$-as extensively discussed before ${ }^{2.6}$ - creating an intermediate $T$ region where FM clusters with random moment-orientations are found. A grid of resistors can be constructed and the effective cluster resistance can be calculated, following standard procedures 6 . Results are in Fig 3 Panel (a) shows the net resistivity $\rho$ vs. $T$, at several $J^{\prime}$ s, with weak $(W \ll J)$ disorder incorporated. For all $J^{\prime} \mathrm{s}$, a fairly sharp peak is found between $T_{C}$ and $T^{*}$ for $\alpha=3$, the exponent that mimics the effect of elastic forces. These $\rho$-profiles are in good agreement with Mn-oxide experiments ${ }^{24}$. Figure 3b illustrates the $W$ dependence of the results. For sufficiently large $W$, the clusters are small and $\rho$ is not enhanced at intermediate temperatures. As $W$ is reduced, the clusters increase in size and the peak in $\rho$ develops 24 . Figure 3, contains $\rho$ vs. $T$, parametric with magnetic fields, for 

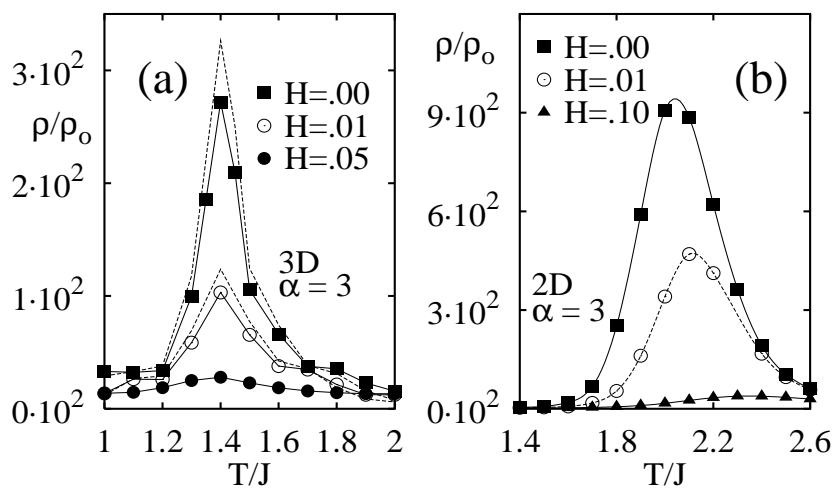

FIG. 4: (a) $\rho / \rho_{0}$ vs. $T$ for the $J-J^{\prime}$ model $\left(J^{\prime} / J=0.23\right.$ and $W / J=0.05)$ with long-range correlated disorder $(\alpha=3)$, in 3D and for the magnetic fields indicated. Solid (dashed) lines are results on $16^{3}\left(32^{3}\right)$ lattices. (b) Same as (a), but on a $64^{2}$ 2D lattice, with $J^{\prime} / J=0.68$ and $W / J=0.1$. Clearly, now both $2 \mathrm{D}$ and $3 \mathrm{D}$ results are quite similar in magnitude.

the case of uncorrelated disorder. In agreement with the introductory discussion, $\rho$ here is two orders of magnitude smaller than with correlated disorder, illustrating the dramatic differences that correlated quenched-disorder causes in the quantitative results. Finally, dominant MC configuration snapshots (Fig 3 d) reveal an intricate cluster arrangement in the $T$ region of interest if $\alpha=3$ - far from the uniformly polarized state at the same $W$ if $\alpha=\infty$ - intuitively justifying the observed high $\rho$ values $^{2,6}$. The proximity of $\alpha=3$ to $\alpha_{c}=2.5$, and the anticipated further effective reduction of $\alpha$ if Coulombic disorder effects were incorporated, lead us to believe that the sub- $\mu$ m clusters reported by Uehara et al. (Ref 5) could in- deed be intrinsic to Mn-oxides.

Although the previous results clearly show that disordercorrelation effects are crucial, only a magnetoresistance estimation can clarify its role in the CMR effect. For this purpose, Fig. 4 contains a 3D/2D resistor-network calculation of $\rho$, with correlated disorder, varying the magnetic field. The peak in the resistivity - in the region between $T_{C}$ and $T^{*}-$ is quite clear and has a similar value (just a factor 3 of difference) in both 3D and 2D. This is a considerable improvement over results with uncorrelated disorder, where the peak resistivity ratios between $2 \mathrm{D}$ and $3 \mathrm{D}$ are as high as 200 or more (see Ref 6 and Fig 3k). The effect of small magnetic fields - that rotate large preformed FM clusters - is now strong in both dimensions of interest, and colossal MR ratios are obtained with minimal tuning of couplings, as shown in Fig 4

Summarizing, here the key role of cooperative effects in the theoretical description of the CMR effect has been unveiled. Cooperation induces correlation in the disorder needed to transform a first-order FM-AF transition into a percolative process. Explicit calculations in toy models for the CMR phenomenon show that the critical dimension of the system is altered by disorder correlation, and when elasticity effects are included magnetoresistance ratios of comparable magnitude are obtained in 2D and 3D. These results remove a conceptual roadblock of previous phase-separation-based theoretical studies of manganites by demonstrating the importance of correlated disorder induced by cooperative strain effects, reaffirming the relevance of clustered states in the description of transition-metal oxides.

This work was supported by the NSF grant DMR-0122523. Conversations with K. Yang, S. L. Cooper, D. Argyriou, K. H. Ahn, A. R. Bishop, D. Khomskii, M. J. Calderón, and A. Millis are gratefully acknowledged.
${ }^{1}$ Colossal Magnetoresistance Oxides, edited by Y. Tokura, Gordon \& Breach, New York, 2000. A. Moreo et al., Science 283, 2034 (1999); Y. Tokura and N. Nagaosa, Science 288, 462 (2000).

2 E. Dagotto, Nanoscale Phase Separation and Colossal Magnetoresistance, Springer-Verlag, Berlin, 2002.

3 S. H. Pan et al., Nature 413, 282 (2001).

${ }^{4}$ E. Dagotto, T. Hotta and A. Moreo, Phys. Rep. 344, 1 (2001).

5 M. Uehara et al., Nature 399, 560 (1999); M. Fäth et al., Science 285, 1540 (1999); Ch. Renner et al., Nature 416, 518 (2002). See also J. Lynn et al., Phys. Rev. Lett. 76, 4046 (1996), and J. De Teresa et al., Nature 386, 256 (1997); D. N. Argyriou et al., Phys. Rev. Lett. 89, 036401 (2002).

6 J. Burgy et al., Phys. Rev. Lett. 87, 277202 (2001).

${ }^{7}$ H. Rho et al., Phys. Rev. Lett. 88, 127401 (2002); C. S. Snow et al., Phys. Rev. Lett. 89, 226401 (2002); G. Alvarez et al., Phys. Rev. Lett. 89, 277202 (2002).

8 Y. Imry and S. K. Ma, Phys. Rev. Lett. 35, 1399 (1975). See also J. F. Fernandez et al., Phys. Rev. Lett. 51, 203 (1983).

9 The authors thank A. Millis for remarking the dimensionality dependence of the results in Ref 6 .

10 D. Akahoshi et al., Phys. Rev. Lett. 90, 177203 (2003).

${ }^{11}$ H. Aliaga et al., cond-mat/0303513
12 Y. Motome et al., cond-mat/0304543

${ }^{13}$ It is interesting to note the close similarities of the cluster shapes with those investigated in J. Burgy et al., Phys. Rev. B67, 014410 (2003) and D. Khomskii and L. Khomskii, Phys. Rev. B67, 052406 (2003).

14 D. I. Khomskii and K. I. Kugel, Europhys. Lett. 55, 208 (2001); Phys. Rev. B67, 134401 (2003).

15 A. R. Bishop et al., Europhys. Lett. 63, 289 (2003); J-X. Zhu et al., Phys. Rev. Lett. 91, 057004 (2003); See also T. Lookman et al., Phys. Rev. B67, 024114 (2003); S. R. Shenoy et al., Phys. Rev. B60, R12537 (1999); K. O. Rasmussen et al., Phys. Rev. Lett. 87, 055704 (2001); K. H. Ahn et al., cond-mat/0207224 A. Bussmann-Holder and A. R. Bishop, Phys. Rev. B56, 5297 (1997).

16 T. Hotta et al., Phys. Rev. B60, R15009 (1999); S. Yunoki et al., Phys. Rev. Lett. 84, 3714 (2000). See also J. A. Verges et al., Phys. Rev. Lett. 88, 136401 (2002).

17 T. Hotta et al., Phys. Rev. Lett. 86, 4922 (2001).

18 C. S. Nelson et al., cond-mat/0303228 and references therein.

19 M. J. Calderón, A. Millis, and K. H. Ahn, cond-mat/0305440 See also K. H. Ahn and A. Millis, Phys. Rev. B64, 115103 (2001).

${ }^{20}$ Kun Yang, Phys. Rev. B67, 092201 (2003). 
21 T. Nattermann, J. Phys. C16, 6407 (1983).

22 T. Hotta et al., Phys. Rev. Lett. 90, 247203 (2003).

23 A. Goldberg and R. Tarjan, J. of the Assoc. for Computing Machinery 35, 921 (1988), and references therein. See also J. Burgy, Ph.D. dissertation, Florida State University, 2003.
24 The $\rho$ reduction as the critical $J^{\prime}=0.25$ is reached in (a) or as $W$ is reduced in (b) is a size effect, which occurs when intrinsic coexisting-cluster sizes become comparable with MC lattices. 